Intensivmed 2010 • 47:552

DOI 10.1007/s00390-010-0190-5

Online publiziert: 29. Oktober 2010

(c) Springer-Verlag 2010

\author{
R.M. Schmid \\ II. Medizinische Klinik, Klinikum rechts der Isar der TU München
}

\title{
Komplikationen bei Leberzirrhose
}

steht jedoch gleichzeitig eine extrasplanchische Vasokonstriktion. Diese bedingt eine verminderte renale Durchblutung mit der Aktivierung des Renin-Angiotensin-Aldosteron-Systems und dadurch eine Funktionseinschränkung der Niere, die als hepatorenales Syndrom bezeichnet wird.

Wichtig ist, dass die Interpretation der Laborwerte bei Patienten mit Leberzirrhose schwierig sein kann. So werden für Serumkreatinin und Serumharnstoff bei Lebererkrankungen häufig niedrigere Werte ermittelt, da Kreatinin vermindert produziert wird und die Muskelmasse häufig reduziert ist. Die Bildung von Aszites bei reduzierter unspezifischer Abwehr begünstigt das Auftreten der spontan bakteriellen Peritonitis, die häufigste spontane Infektion bei Patienten mit Leberzirrhose und Aszites. Da die klinische Präsentation einer spontan bakteriellen Peritonitis meist symptomarm ist, kann diese leicht übersehen werden. Entscheidend ist es, an diese Möglichkeit zu denken.

\section{- Die Lebertransplantation ist die einzige kurative Therapie der Leberzirrhose.}

Dies betrifft aber nur einen kleinen Teil der Patienten. Viele Patienten sterben, da für sie nicht rechtzeitig ein Organ zur Verfügung steht. Ideal wäre ein Leberersatzverfahren in Analogie zur Dialyse bei Patienten mit Nierenversagen. Ein solches System würde bei akut-auf-chronischem Leberversagen zum Einsatz kommen. Obwohl kleine Studien positive Resultate er- bracht haben, zeigen größere Studien keinen Überlebensvorteil für die Patienten. Die derzeitigen Verfahren können die hepatische Enzephalopathie verbessern, nicht jedoch wesentliche Organschäden bei Multiorganversagen.

Das zunehmende Verständnis um die Pathophysiologie der Zirrhose und der damit verbundenen Komplikationen hat in den letzten Jahren zu einer genaueren Diagnostik, einem verbesserten Management und infolgedessen zu besserer Lebensqualität und längerer Lebenszeit für Patienten mit Leberzirrhose geführt. Von zentraler Wichtigkeit bleibt aber weiterhin das rechtzeitige Erkennen der Komplikationen der Leberzirrhose, da deren Management eine hohe Kompetenz und die Koordination verschiedener Fachdisziplinen erforderlich macht.

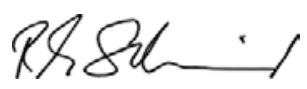

Prof. Dr. Roland M. Schmid

\section{Korrespondenzadresse Prof. Dr. R.M. Schmid}

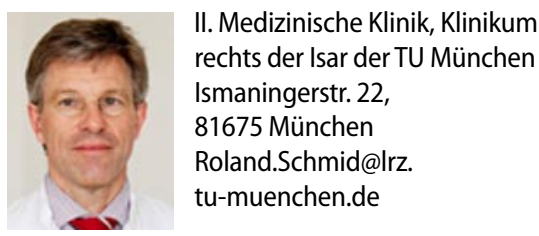

